\title{
Quantum interference in the classically forbidden region: A parametric oscillator
}

\author{
M. Marthaler ${ }^{1,2}$ and M. I. Dykman ${ }^{1}$ \\ ${ }^{1}$ Department of Physics and Astronomy, Michigan State University, East Lansing, MI 48824, USA \\ ${ }^{2}$ Institut für Theoretische Festkörperphysik and DFG-Center for Functional Nanostructures (CFN), \\ Universität Karlsruhe, D-76128 Karlsruhe, Germany
}

(Dated: February 6, 2008)

\begin{abstract}
We study tunneling between period-2 states of a parametrically modulated oscillator. The tunneling matrix element is shown to oscillate with the varying frequency of the modulating field. The effect is due to spatial oscillations of the wave function and the related interference in the classically forbidden region. The oscillations emerge already in the ground state of the oscillator Hamiltonian in the rotating frame.
\end{abstract}

PACS numbers: 74.50.+r, 03.65.Xp, 05.45.-a, 05.60.Gg

Nonlinear micro- and mesoscopic vibrational systems have attracted much interest in recent years. In such systems damping is often weak, and even a comparatively small resonant field can lead to bistability, i.e., to coexistence of forced vibrations with different phases and/or amplitudes. Quantum and classical fluctuations cause transitions between coexisting vibrational states. The transitions are not described by the conventional theory of metastable decay, because the states are periodic in time and the systems lack detailed balance. Experimentally, classical transition rates have been studied for such diverse vibrational systems as modulated trapped electrons [1], Josephson junctions [2], nano- and micromechanical oscillators [3, 4, 5], and trapped atoms [6], and the results are in agreement with theory $[7,8]$.

Currently much experimental effort is being put into reaching the quantum regime [9, 10]. In this regime tunneling between coexisting classically stable periodic states should become important, for weak dissipation. It was first studied for a resonantly driven oscillator, where a semiclassical analysis [1] made it possible to find the tunneling exponent in a broad parameter range [12].

Tunneling is particularly interesting for a parametrically modulated oscillator. Here, the coexisting classical periodic states have period $2 \tau_{F}$, where $\tau_{F}$ is the modulation period. Such period-2 states are identical except that the vibrations are shifted in phase by $\pi$. Therefore the corresponding quantum states (Floquet states) are degenerate. Tunneling should lift this degeneracy, as for a particle in a symmetric static double-well potential. Earlier the tunneling matrix element was found [13] for modulation at exactly twice the oscillator eigenfrequency $\omega_{0}$. Recently the tunneling exponent was obtained in a general case where the modulation frequency $\omega_{F}=2 \pi / \tau_{F}$ is close to $2 \omega_{0}[14]$

In this paper we show that tunneling between period-2 states of a parametrically modulated oscillator displays unexpected features. We find that the tunneling matrix element oscillates with varying $\omega_{F}-2 \omega_{0}$, periodically passing through zero. These oscillations are accompanied by, and are due to spatial oscillations of the wave function in the classically forbidden region.

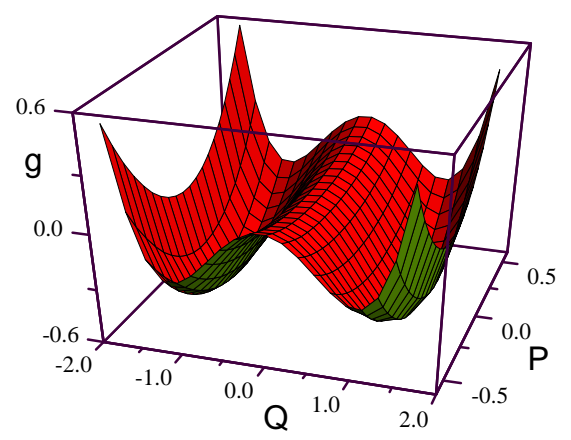

FIG. 1: (Color online) The scaled effective Hamiltonian of the oscillator in the rotating frame $g(Q, P)$, Eq. (5), for $\mu=0.5$. The minima of $g(Q, P)$ correspond to the period-2 vibrations. The eigenvalues of $\hat{g}$ give scaled oscillator quasienergies.

For resonant modulation, $\left|\omega_{F}-2 \omega_{0}\right| \ll \omega_{F}$, and for a small amplitude of the modulating field $F$ the oscillator dynamics is well described by the rotating wave approximation (RWA) [15]. The scaled RWA Hamiltonian $\hat{g}$ as a function of the oscillator coordinate $Q$ and momentum $P$ in the rotating frame is independent of time. In a broad parameter range it has a symmetric double-well form shown in Fig. 1. The minima correspond to the classical period-2 states, in the presence of weak dissipation. Respectively, of utmost interest are tunneling transitions between the lowest single-well quantum states of $\hat{g}$.

A simple model of a nonlinear oscillator that describes many experimental systems, cf. Refs. 1, 2, 3, 3, 4, ,5, 6, is a Duffing oscillator. The Hamiltonian of a parametrically modulated Duffing oscillator has the form

$$
H_{0}=\frac{1}{2} p^{2}+\frac{1}{2}\left(\omega_{0}^{2}+F \cos \omega_{F} t\right) q^{2}+\frac{1}{4} \gamma q^{4} .
$$

For $\omega_{F}$ close to $2 \omega_{0}$ and for comparatively small $F$,

$$
\delta \omega=\frac{1}{2} \omega_{F}-\omega_{0}, \quad|\delta \omega| \ll \omega_{0}, \quad F \ll \omega_{0}^{2},
$$


even where the oscillator becomes bistable its nonlinearity remains relatively small, $\left|\gamma\left\langle q^{2}\right\rangle\right| \ll \omega_{0}^{2}$. For concreteness we set $\gamma>0$; the results for $\gamma<0$ can be obtained by replacing $\delta \omega \rightarrow-\delta \omega$ in the final expressions.

To describe a weakly nonlinear oscillator it is convenient to make a canonical transformation from $q$ and $p$ to the slowly varying coordinate $Q$ and momentum $P$,

$$
\begin{aligned}
U^{\dagger} q U & =C_{\mathrm{par}}\left[P \cos \left(\omega_{F} t / 2\right)-Q \sin \left(\omega_{F} t / 2\right)\right] \\
U^{\dagger} p U & =-C_{\mathrm{par}} \omega_{F}\left[P \sin \left(\omega_{F} t / 2\right)+Q \cos \left(\omega_{F} t / 2\right)\right] / 2,
\end{aligned}
$$

where $C_{\mathrm{par}}=(2 F / 3 \gamma)^{1 / 2}$ and

$$
[P, Q]=-i \lambda, \quad \lambda=3 \gamma \hbar / F \omega_{F} .
$$

The dimensionless parameter $\lambda$ plays the role of $\hbar$ in quantum dynamics in the rotating frame [14].

The transformed oscillator Hamiltonian has the form $\left(F^{2} / 6 \gamma\right) \hat{g}$, where $\hat{g} \equiv g(Q, P)$,

$g(Q, P)=\frac{1}{4}\left(P^{2}+Q^{2}\right)^{2}+\frac{1}{2}(1-\mu) P^{2}-\frac{1}{2}(1+\mu) Q^{2}(5)$

[we use here a more conventional notation $g(Q, P)$ instead of $g(P, Q)$ used in Ref. 14]. The terms $\propto$ $\exp \left( \pm i n \omega_{F} t\right)$ with $n \geq 1$ in $\hat{g}$ have been disregarded.

The time-independent operator $\hat{g}$ is the scaled oscillator Hamiltonian in the rotating frame. Its eigenvalues multiplied by $F^{2} / 6 \gamma$ give oscillator quasienergies, or Floquet eigenvalues. Formally, $\hat{g}$ is a Hamiltonian of an auxiliary stationary system with variables $Q, P$, and the eigenvalues of $\hat{g}$ give the energies of this system. The operator $\hat{g}$ depends on one parameter

$$
\mu=2 \omega_{F} \delta \omega / F .
$$

For $\mu>-1, g(Q, P)$ has two minima located at $P=0$, $Q= \pm(\mu+1)^{1 / 2}$. For $\mu \leq 1$ the minima are separated by a saddle at $P=Q=0$, as shown in Fig. 1. When friction is taken into account, the minima become stable states of period-2 vibrations. The function $g(Q, P)$ is symmetric as a consequence of the time translation symmetry: the change $(P, Q) \rightarrow(-P,-Q)$ corresponds to shifting time in Eq. (3) by the modulation period $\tau_{F}$.

We assume the effective Planck constant $\lambda$ to be the small parameter of the theory, $\lambda \ll 1$. Then the low-lying eigenvalues of $\hat{g}$ form doublets. Splitting of the doublets is due to tunneling between the wells of $g(Q, P)$. Since $g(Q, P)=g(-Q,-P)$ is symmetric, the problem of level splitting seems to be similar to the standard problem of level splitting in a double-well potential [16]. As in this latter case, we will analyze it in the WKB approximation.

The major distinction of the present problem comes from the difference between the structure of $g(Q, P)$ and the Hamiltonian considered in Ref. 16. The momentum $P(Q ; g)$ as given by equation $g(Q, P)=g$ has 4 branches, with both real and imaginary parts in the classically forbidden region of $Q$. This leads to new features of tunneling and requires a modification of the method [16].
We will consider splitting $\delta g$ of the two lowest eigenvalues of $\hat{g}$. Because of the symmetry, the corresponding wave functions $\psi_{ \pm}(Q)$ are

$$
\psi_{ \pm}(Q)=\frac{1}{\sqrt{2}}\left[\psi_{l}(Q) \pm \psi_{l}(-Q)\right]
$$

where $\psi_{l}(Q)$ is the "single-well" wave function of the left well of $g(Q, P)$ in Fig. 1 It is maximal at the bottom of the well $Q_{l 0}=-(1+\mu)^{1 / 2}$ and decays away from the well. To the leading order in $\lambda$, the corresponding lowest eigenvalue of $\hat{g}$ is $g_{\min }+g_{q}$, where $g_{\min }=-(1+\mu)^{2} / 4$ is the minimum of $g(Q, P)$ and $g_{q}=\lambda(\mu+1)^{1 / 2}$ is the quantum correction.

The wave function $\psi_{l}(Q)$ is particularly simple for $\mu<$ 0 . In the classically forbidden region between the wells, $|Q|<\left|Q_{l 0}\right|$, it has the form

$$
\psi_{l}=C\left[-i \partial_{P} g\right]^{-1 / 2} \exp \left[i S_{0}(Q) / \lambda\right]
$$

where $S_{0}(Q)$ is given by the equation $g\left(Q, \partial_{Q} S_{0}\right)=$ $g_{\min }+g_{q}$,

$$
\begin{aligned}
S_{0}(Q) & =\int_{Q_{l 0}+L_{q}}^{Q} P_{-}\left(Q^{\prime}\right) d Q^{\prime}, \\
P_{ \pm}(Q)= & i\left[1+Q^{2}-\mu \pm 2\left(Q^{2}-\tilde{\mu}\right)^{1 / 2}\right]^{1 / 2} \\
\tilde{\mu}=\mu-g_{q}, & L_{q}=\lambda / g_{q}^{1 / 2} \equiv \lambda^{1 / 2}(\mu+1)^{-1 / 4} .
\end{aligned}
$$

We keep here only the contribution from the branch $P_{-}(Q)$, because $P_{-}(Q)$ is zero on the boundary of the classically forbidden range $Q_{l 0}+L_{q}$. For $-\mu \gg \lambda$ and $\left|Q_{l 0}+L_{q}\right|>|Q|$ the action $S_{0}(Q)$ is purely imaginary. The wave function $\psi_{l}(Q)$ monotonically decays with increasing $Q$.

The prefactor in the wave function (8) is determined by the complex classical speed of the oscillator

$$
\partial_{P} g=2 P_{-}(Q)\left(Q^{2}-\tilde{\mu}\right)^{1 / 2} .
$$

The normalization constant $C$ in Eq. (8),

$$
C=[(\mu+1) / \pi]^{1 / 4} \exp (-1 / 4),
$$

is obtained by matching, in the range $L_{q} \ll Q-Q_{l 0} \ll$ $\left|Q_{l 0}\right|$, Eq. (8) to the tail of the Gaussian peak of $\psi_{l}(Q)$, which is centered at $Q_{l 0}$.

We are most interested in the parameter range $\mu \gg \lambda$ where tunneling displays unusual behavior. For such $\mu$ the momentum $P_{-}(Q)$ becomes complex in the range $|Q|<\tilde{\mu}$. This means that the decay of the wave function is accompanied by oscillations. To correctly describe them we had to keep corrections $\propto g_{q}$ in Eq. (9).

We first rewrite Eq. (9) in the form

$$
P_{-}(Q) \approx i\left[1-\left(Q^{2}-\tilde{\mu}\right)^{1 / 2}-\frac{g_{q} / 2}{1-\left(Q^{2}-\tilde{\mu}\right)^{1 / 2}}\right] .
$$


Eq. (12) applies for $Q-Q_{l 0} \gg L_{q}$. It is seen that $P_{-}(Q)$ has two branching points inside the classically forbidden region. The closest to $Q_{l 0}$ is the point $Q_{b r}=-\tilde{\mu}^{1 / 2}$. The WKB approximation breaks down for small $Q+\tilde{\mu}^{1 / 2}$. The wave function in this region can be shown to be proportional to Airy function $\operatorname{Ai}\left[-\left(Q+\tilde{\mu}^{1 / 2}\right)\left(2 \tilde{\mu}^{1 / 2} / \lambda^{2}\right)^{1 / 3}\right]$. Therefore $\psi_{l}$ oscillates with $Q$ for positive $Q+\tilde{\mu}^{1 / 2}$.

In contrast to the standard WKB theory of the turning point, the prefactor in $\psi_{l}$ contains two factors that experience branching at $-\tilde{\mu}^{1 / 2}$, see Eqs. (8), (10). The full solution in the oscillation region can be obtained by going around $-\tilde{\mu}^{1 / 2}$ in the complex plane following the prescription [16]. For $\lambda^{2 / 3} \ll Q+\tilde{\mu}^{1 / 2}$ it gives

$$
\begin{aligned}
& \psi_{l} \approx 2 C\left|\partial_{P} g\right|^{-1 / 2} \exp \left[-\operatorname{Im} S_{0}(Q) / \lambda\right] \cos \Phi(Q), \\
& \Phi(Q)=\Phi_{1}(Q)+\Phi_{2}(Q) .
\end{aligned}
$$

The term $\operatorname{Im} S_{0}(Q)$ in the amplitude of the wave function (13) is determined by Eq. (9). The phase $\Phi(Q)$ has two terms. The term $\Phi_{1}(Q)$ comes from the exponential factor in the WKB wave function (8),

$$
\Phi_{1}(Q)=\lambda^{-1} \int_{-\tilde{\mu}^{1 / 2}}^{Q} \operatorname{Re} P_{-}(Q) d Q
$$

where $\operatorname{Re} P_{-}(Q)$ is given by Eq. (12) in which we set $\left(Q^{2}-\tilde{\mu}\right)^{1 / 2} \rightarrow i\left(\tilde{\mu}-Q^{2}\right)^{1 / 2}$; therefore $\operatorname{Re} P_{-}(Q)>0$. It is simple to write $\Phi_{1}$ and $\operatorname{Im} S_{0}(Q)$ in explicit form.

The term $\Phi_{2}(Q)$ in Eq. (13) comes from the prefactor in $\psi_{l}(Q)$, Eq. (8),

$$
\Phi_{2}(Q) \approx \frac{1}{2} \arcsin \left(\frac{\mu-Q^{2}}{1+\mu-Q^{2}}\right)^{1 / 2}-\frac{\pi}{4} .
$$

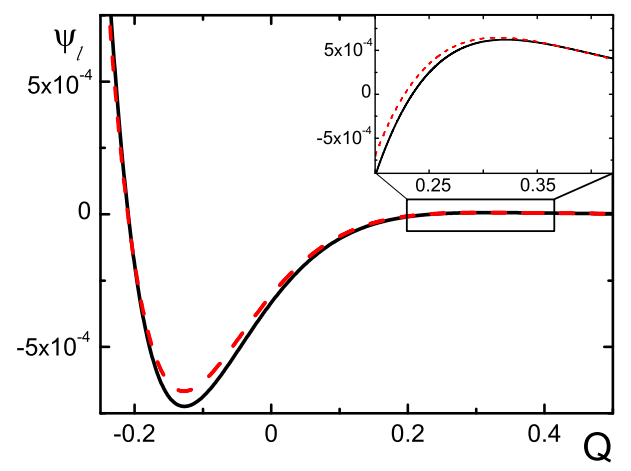

FIG. 2: (Color online) The wave function of the ground state in the left well $\psi_{l}(Q)$ in the oscillation region for $\lambda=0.09$ and $\mu=0.5$. The solid line shows explicit expressions (13)-(15), the dashed line shows numerical results. Inset: $\psi_{l}(Q)$ near its second zero with higher resolution.

Decay and oscillations of the wave function described by Eq. (13) are compared in Fig. 2 with the results of a numerical solution of the Schrödinger equation $\hat{g} \psi=g \psi$. The left-well wave function was obtained numerically as a sum of the two lowest-eigenvalues solutions, cf. Eq. (7). In this calculation the basis of 120 oscillator Fock states was used. A good agreement between analytical and numerical results is seen already for not too small $\lambda=0.09$.

The above solution allows us to find the tunnel splitting $\delta g=g_{-}-g_{+}$of the symmetric and antisymmetric states (7). Following the standard approach for a symmetric double-well potential [16] we multiply the Schrödinger equations for the involved states $\hat{g} \psi_{l}=g_{l} \psi_{l}$ and $\hat{g} \psi_{ \pm}=g_{ \pm} \psi_{ \pm}$by $\psi_{ \pm}^{*}$ and $\psi_{l}^{*}$, respectively, integrate over $Q$ from $-\infty$ to 0 and subtract the results. This gives

$$
\begin{array}{r}
\delta g=-\lambda^{2}\left\{2(1-\mu) \psi_{l}(0) \psi_{l}^{\prime}(0)\right. \\
\left.-\lambda^{2}\left[\psi_{l}(0) \psi_{l}^{\prime \prime \prime}(0)+\psi_{l}^{\prime}(0) \psi_{l}^{\prime \prime}(0)\right]\right\}
\end{array}
$$

or, with account taken of Eq. (13),

$$
\begin{aligned}
\delta g= & \frac{16 \lambda^{1 / 2}(\mu+1)^{5 / 4}}{(\pi \mu)^{1 / 2}} e^{-A / \lambda} \cos \left[2 \Phi_{1}(0)\right], \\
A= & (\mu+1)^{1 / 2}+\mu \ln \left(\mu^{-1 / 2}\left[1+(\mu+1)^{1 / 2}\right]\right), \\
& 2 \Phi_{1}(0)=\pi\left(\mu \lambda^{-1}-1\right) / 2 \quad(\mu \gg \lambda) .
\end{aligned}
$$

Clearly, $\delta g$ may be positive or negative, that is, the symmetric state may have a lower or higher quasienergy than the antisymmetric state.

The dimensional splitting $\left(F^{2} / 6 \gamma\right)|\delta g|$ gives twice the matrix element of tunneling between period-2 states of the oscillator. This matrix element has an exponential factor $\exp (-A / \lambda)$ 14]. In addition, it contains a factor oscillating as a function of the scaled frequency detuning $\mu / \lambda=6 \omega_{F}^{2}\left(\omega_{F}-2 \omega_{0}\right) / 3 \gamma \hbar$. The oscillation period is $\Delta(\mu / \lambda)=4$. These oscillations are shown in Fig. 3 .

The oscillations of $\delta g$ result from the wave function oscillations in the classically forbidden region. This can be seen from the analysis of $\psi_{l}(Q)$ near the positive- $Q$ boundary of the oscillation region, $Q=\tilde{\mu}^{1 / 2}$. The wave function for $Q-\tilde{\mu}^{1 / 2} \gg \lambda$ is a combination of the WKB waves with imaginary momenta $P_{ \pm}(Q) \approx i\left[1 \pm\left(Q^{2}-\right.\right.$ $\tilde{\mu})^{1 / 2}$. The coefficients in this combination can be found in a standard way [16]. They are determined by the phase $\Phi\left(\tilde{\mu}^{1 / 2}\right)$. Only the wave with $P_{-}(Q)$ contributes to the tunneling amplitude, since $P_{+}$remains imaginary in the right well of $g(Q, P)$. For $\Phi\left(\tilde{\mu}^{1 / 2}\right)=(4 n-3) \pi / 4$ this wave has zero amplitude, leading to $\delta g=0$. By noting that $\Phi\left(\tilde{\mu}^{1 / 2}\right)=2 \Phi_{1}(0)-\pi / 4$, we immediately obtain from Eq. (16) that $\delta g=0$ for $\mu=2 n \lambda$ with integer $n$, in agreement with Fig. 3

The occurrence of spatial oscillations of the ground state wave function of the scaled Hamiltonian $\hat{g}$ does not contradict the oscillation theorem, because $\hat{g}$ is not a sum of the kinetic and potential energies and is quartic in $P$. The motion described by the Hamiltonian $g(Q, P)$ is classically integrable. Respectively, the quantum problem is 


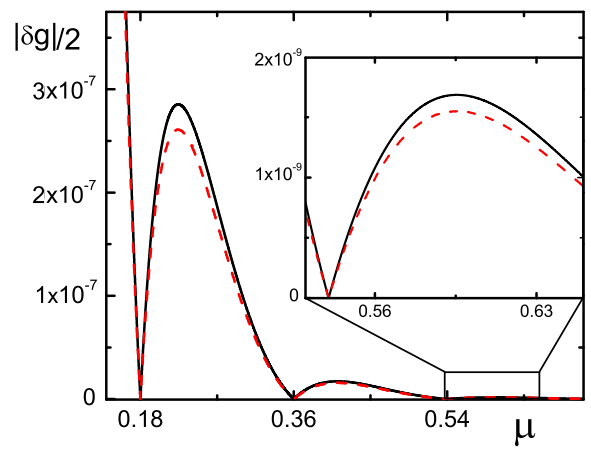

FIG. 3: (Color online) Scaled matrix element of tunneling between period-2 states as a function of the scaled detuning of the modulation frequency from twice the oscillator eigenfrequency. The solid lines show explicit expression (16), the dashed lines show the result of numerical calculations. Inset: a higher-resolution plot of $|\delta g| / 2$ vs. $\mu$ near the zero of $\delta g$ at $\mu=6 \lambda$. The data refer to $\lambda=0.09$.

different from dynamical tunneling in classically chaotic systems [17, 18, 19]; the effect we discuss has not been considered for such systems, to the best of our knowledge.

The effect is also qualitatively different from photonassisted/suppressed tunneling in systems with stationary double-well potentials: our oscillator has a single-well potential, the bistability is a consequence of resonant modulation, and the Hamiltonian $\hat{g}$ is independent of time. At the same time there is a remote similarity between the oscillations of the tunneling matrix element for period-2 states and for electron states in a double-well potential in a quantizing magnetic field [20]. However, not only is the physics different, but our approach is also different from that in Ref. 20; in particular, it makes it possible to find $\delta g$ analytically. The approach can be also extended to a resonantly driven Duffing oscillator, where the RWA Hamiltonian has a structure similar to Eq. (5) 7, 12].

Tunnel splitting can be observed by preparing the system in one of the period-2 states and by studying interstate oscillations, cf. Refs. 18, 19. This requires that the tunneling rate $(\delta \omega / 2 \mu \lambda)|\delta g|$ exceed $\omega_{F} / 4 \mathcal{Q}$, where $\mathcal{Q}$ is the oscillator quality factor. The splitting sharply increases with increasing $\lambda$. It will be shown separately that for comparatively large $\lambda$ (but still for $|\delta g| \ll g_{q}$ ) the RWA applies and relaxation remains small provided $\delta g^{2} \gg C_{\lambda} / \mathcal{Q}$ with $C_{\lambda} \lesssim 1$. Our RWA numerical results indicate that $\delta g$ still oscillates with $\mu$ for $\lambda=0.25-0.3$ and is well described by Eq. (16) for $\mu \gtrsim 2 \lambda$. The local peak of $|\delta g|$ for $\lambda=0.3$ and the characteristic extremum of $d^{2} \delta g / d \mu^{2}$ for $\lambda=0.25$ occur where $|\delta g| \approx 0.01$. Such $\delta g$ may be large enough for detecting the effect in modulated Josephson junctions where $\mathcal{Q}=2360$ has been reached in the range of bistability [10].

In conclusion, we used the WKB approximation to study the wave functions of the period-2 states of a parametrically modulated oscillator. We showed that these wave functions can display spatial oscillations in the classically forbidden region, in the rotating frame. These oscillations lead to oscillations of the matrix element of tunneling between the period-2 states with the varying frequency of the modulating field.

We are grateful to V.N. Smelyanskiy and F. Wilhelm for stimulating interactions. This research was supported in part by the NSF through grant No. PHY-0555346.

[1] L. J. Lapidus, D. Enzer, and G. Gabrielse, Phys. Rev. Lett. 83, 899 (1999).

[2] I. Siddiqi, R. Vijay, F. Pierre, C. M. Wilson, L. Frunzio, M. Metcalfe, C. Rigetti, R. J. Schoelkopf, M. H. Devoret, D. Vion, et al., Phys. Rev. Lett. 94, 027005 (2005).

[3] J. S. Aldridge and A. N. Cleland, Phys. Rev. Lett. 94, 156403 (2005).

[4] R. Almog, S. Zaitsev, O. Shtempluck, and E. Buks, Appl. Phys. Lett. 90, 013508 (2007).

[5] C. Stambaugh and H. B. Chan, Phys. Rev. B 73, 172302 (2006).

[6] K. Kim, M. S. Heo, K. H. Lee, H. J. Ha, K. Jang, H. R. Noh, and W. Jhe, Phys. Rev. A 72, 053402 (2005).

[7] M. I. Dykman and M. A. Krivoglaz, Zh. Eksper. Teor. Fiz. 77, 60 (1979).

[8] M. I. Dykman, C. M. Maloney, V. N. Smelyanskiy, and M. Silverstein, Phys. Rev. E 57, 5202 (1998).

[9] K. C. Schwab and M. L. Roukes, Phys. Today 58, 36 (2005).

[10] E. Boaknin, V. E. Manucharyan, S. Fissette, M. Metcalfe, L. Frunzio, R. Vijay, I. Siddiqi, A. Wallraff, R. J. Schoelkopf, and M. H. Devoret, cond-mat/0702445 (2007).

[11] V. N. Sazonov and V. I. Finkelstein, Doklady Akad. Nauk SSSR 231, 78 (1976).

[12] A. P. Dmitriev and M. I. Dyakonov, Zh. Eksper. Teor. Fiz. 90, 1430 (1986).

[13] B. Wielinga and G. J. Milburn, Phys. Rev. A 48, 2494 (1993).

[14] M. Marthaler and M. I. Dykman, Phys. Rev. A 73, 042108 (2006).

[15] L. D. Landau and E. M. Lifshitz, Mechanics (Elsevier, Amsterdam, 2004), 3rd ed.

[16] L. D. Landau and E. M. Lifshitz, Quantum mechanics. Non-relativistic theory (Butterworth-Heinemann, Oxford, 1981), 3rd ed.

[17] S. Tomsovic and D. Ullmo, Phys. Rev. E 50, 145 (1994).

[18] W. K. Hensinger, H. Haffer, A. Browaeys, N. R. Heckenberg, K. Helmerson, C. McKenzie, G. J. Milburn, W. D. Phillips, S. L. Rolston, H. Rubinsztein-Dunlop, et al., Nature 412, 52 (2001).

[19] D. A. Steck, W. H. Oskay, and M. G. Raizen, Science 293, 274 (2001).

[20] J. K. Jain and S. Kivelson, Phys. Rev. B 37, 4111 (1988). 